\title{
أثر تطور القطاع المالي علي النمو الاقتصادى في دولة الكويت
}

\author{
نادر صالح علي مصلح عايض \\ طالب دكتوراه بمعهد البحوث والدراسات الأفريقية - جامعة أسوان \\ محمد عبد الحفيظ محما \\ أستاذ الاقتصاد الزراعي وعميد كلية الزراعة - جامعة سوهاج \\ حسن موسي رضوان \\ مدرس الاقتصاد الزراعي بالمعهد العالي للتعاون والإرشاد الزراعي بأسيوط
}


استهدفت الدراسة التعرف علي مفاهيم التطور المالي وعلاقته بالنمو الاقتصادي، ودراسة أثر التطور المالي في النمو الاقتصادي لدولة الكويت، واعتمدت الدراسة علي القياسية بين نصيب الفرد من الناتج المحلي الآجمالي كمتغي تابع، بينما المتغيرات

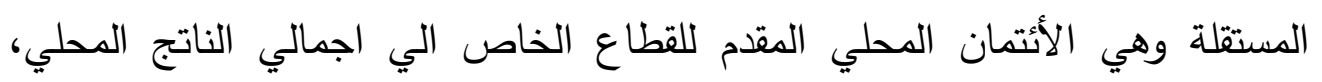
العرض النقدي الموسع الي إجمالي الناتج المحلي، صافي الاستثمار المحلي، النفقات

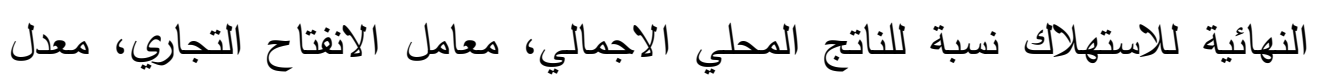
التضخم. وتوصلت الدراسة ان هناك علاقة طردية بين كل من معامل الانفتاح التجاري

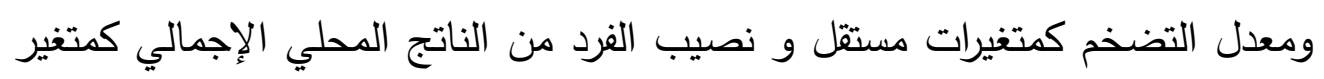
تابع ، حيث أن زيادة كل من معامل الانفتاح التجاري بوحدة واحدة يؤدي الي زيادة

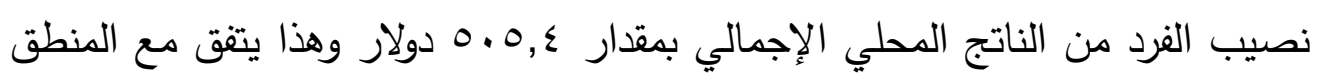
الاقتصادي، في حين يرتط معدل التضخم بعلاقة طرديا مع نصيب الفرد الناتج المحلي الإجمالي حيث ان زيادة معدل التضخم بوحدة واحد يؤدي الي زيادة نصيب الفرد الناتج المحلي الإجمالي بمقدار T ـ ب ب دولار وهذا لايتقق مع المنطق الاقتصادي .بينما لم تثبت معنوية كل من الائتمان المحلي المقدم إلى القطاع الخاص نسبة إلى الناتج الإجمالي المحلي و المعروض النقدي نسبة إلى اجمالي الناتج و النفقات النهائية للاستهلاك نسبة إلى الناتج الإجمالي المحلي. الكلمات الإفتتاحية: القطاع المالى - النمو الإقتصادى - الكويت.

\section{Abstract}

The Impact of The Development of The Financial Sector on The Economic Growth in the State of Kuwait

The study aimed to identify the concepts of financial development and its relationship to economic growth, and to study the effect of financial development on the economic growth of the State of Kuwait. Monetary expanded to GDP, net domestic 
investment, final expenditures for consumption relative to GDP, trade openness coefficient, inflation rate. The study found that there is a positive relationship between the trade openness coefficient and the inflation rate as independent variables and the per capita GDP as a dependent variable, as increasing each of the trade openness coefficient by one unit leads to an increase in the per capita GDP by 505.4 dollars. This is consistent with the economic logic, while the rate of inflation is directly related to the per capita GDP, as an increase in the rate of inflation by one unit leads to an increase in the per capita GDP by $\$ 2606$, and this is not consistent with the economic logic. Provided to the private sector relative to GDP and money supply relative to total product and final expenditures of consumption relative to GDP.

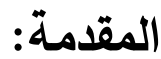

يعتبر النمو الاقتصادي أحد أهم المؤشرات الاقتصادية التي شغلت حيز كبير

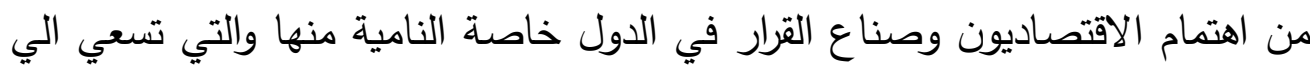

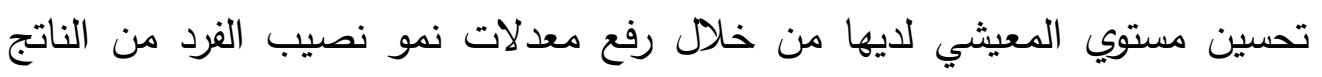

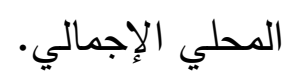

وفي السنوات الأخيرة برزت العديد من النماذج النظرية التي تؤسس لوجود

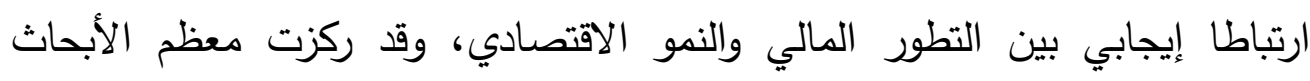

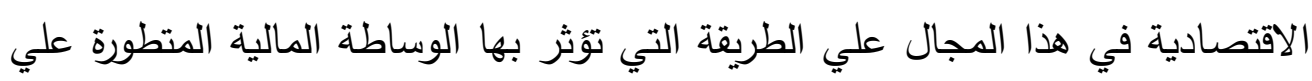

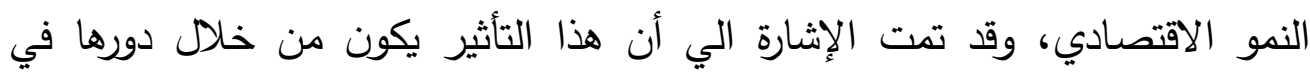

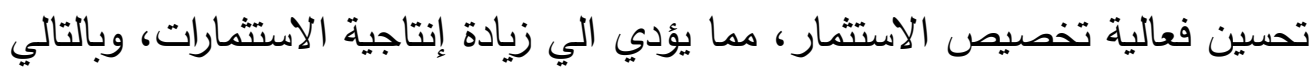

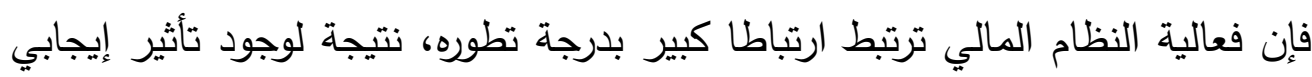

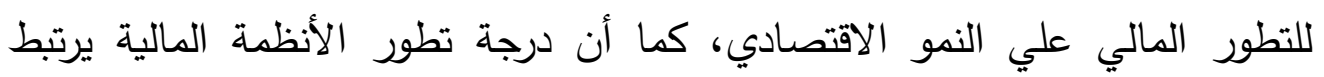

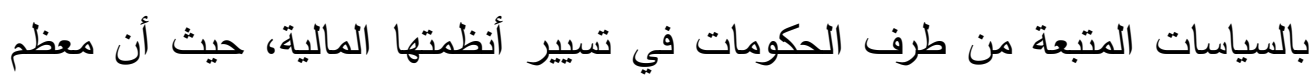

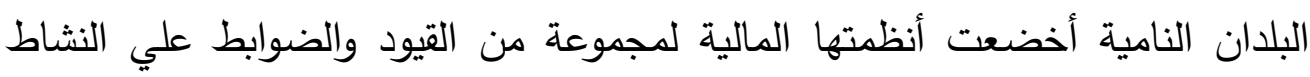

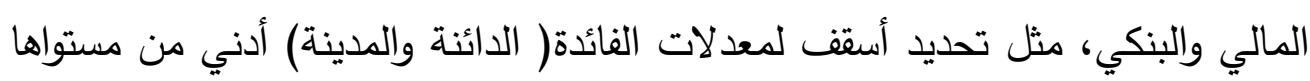

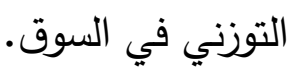




\section{مشكلة الاراسة:}

رغم ما حققته دولة الكويت من زيادة في إجمالي نواتجها الكلية، والتطور المذهل

في أسواق المال وتطور أنشطة القطاع المالي والمصرفي والنقدي، الإ أنها تواجه العديد من التحديات الاقتصادية في سبيل تحقيقها لمعدلات معقولة من التتمية الاقتصادية والاجتماعية، ومن تلك التحديات انخفاض ومحدودية تأثير القطاع المالي في نمو واستدامة قطاعات الاقتصاد الحقيقي، ومشكلات أخري لها علاقة بالبيئة وبمؤشرات

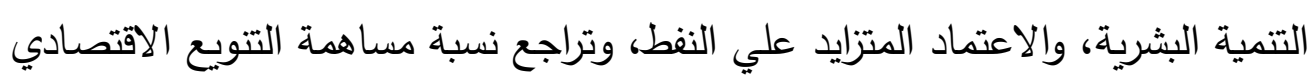
كنسبة من الناتج غير النفطي في دولة الكويت.

\section{فروض الدارسة:}

1- هناك علاقة بين التطور المالي والنمو الاقتصادي في الكويت.

r- يوجد علاقة بين التسهيلات الائتمانية المقدمة الي القطاع الخاص والنمو

$$
\text { الاقتصادي في الكويت. }
$$

r- تحديد اتجاه العلاقة بين التطور المالي والنمو الاقتصادي.

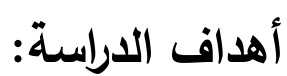

1-تحليل دور التطور في النظام المالي علي النمو الاقتصادي في الكويت. r-تقدير نموذج اقتصادي يبين العلاقة بين مؤشرات التطور المالي والنمو الاقتصادي.

r-قياس أثر التطور في النظام المصرفي علي النمو الاقتصادي في الكويت. مصادر البيانات:

اعتمد الدراسة علي البيانات الرسمية الصادرة عن تقارير البنك الدولي، ومؤسسة

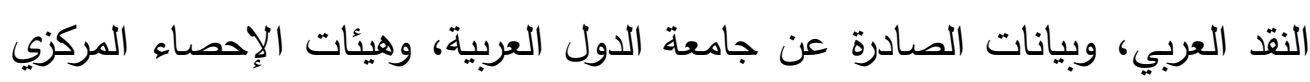
لدولة الكويت، بالآضافة الي الدراسات السابقة والدوريات ذات الصلة بموضوع الدراسة. 


\section{المطلب الاول: تعريف النظام المالي و مكوناته: اولا- تعريف النظام المالى:}

يعبر النظام المالي عن شبكة من الأسواق المالية والمؤسسات المالية ورجال

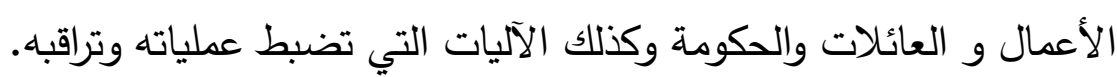
ويتكفل النظام المالي بتوفير الإحتياجات المالية لدختلف المتعاملين الإقتصاديين

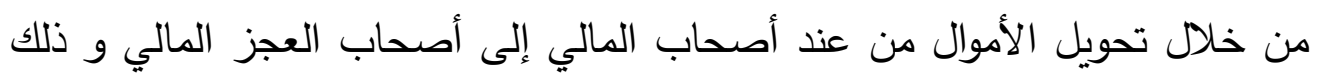

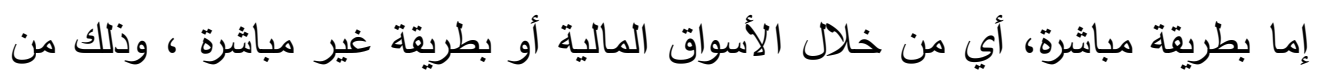
خلال البنوك و المؤسسات المالية المختلفة.

تتوقف فعالية النظام المالي على قدرته في تعبئة الإدخار وضمان أفضل التخصيصات للموارد المالية المتاحة في الإقتصاد.

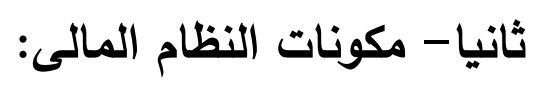
يمكن تقسيم النظام المالي إلى فئتين من المتعاملين: 1- مستخدمو الخدمات المالية ( الوحدات الإستهلاكية، الوحدات الإنتاجية، القطاع الحكومي، القطاع الأجنبي).

أ- الوحدات الإستهلاكية ( قطاع الاستهلاك ) : تتمثل في العائلات والأفراد و

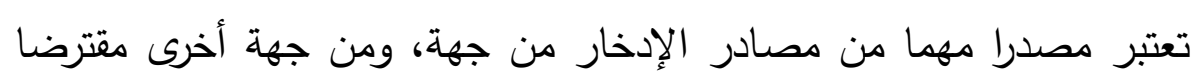
للأموال حيث تتفق من دخلها الجاري على سلع و خدمات الإستهلاك الجاري،

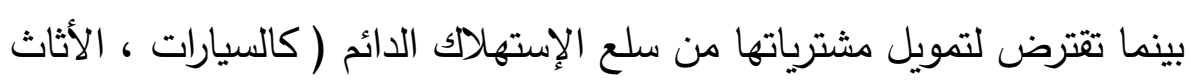
، الأبنية....).

ب- الوحدات الإنتاجية ( القطاع الإنتاجي )_: تعتبر كذلك مصدرا من مصادر الإدخار ولكن ليس بالأهمية الكبرى التي تحتلها الوحدات الإستهلاكية.

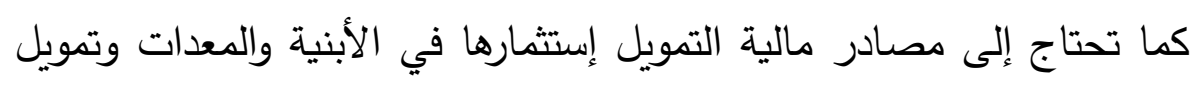
مشترياتها من المواد الأولية أو الوسيطة التي تحتاجها في العملية الإنتاجية. 
ج- القطاع الحكومي : بالإضافة إلى تعامل الدكومة مع القطاع المالي فهي تقوم بتنظيم و ضبط عملياته و تراقب النظام المصرفي كما تستخدم النظام

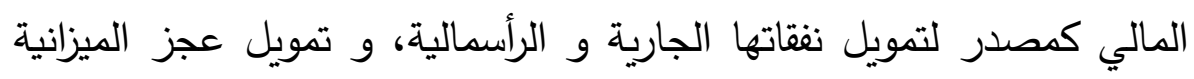
من خلال بيع أوراق مالية للجمهور ( سندات حكومية، أذنات الخزينة).

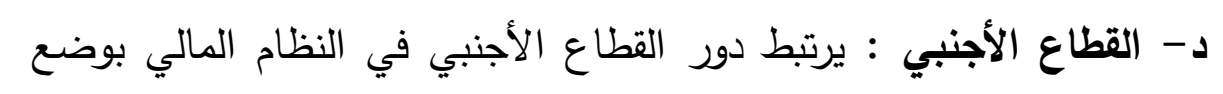

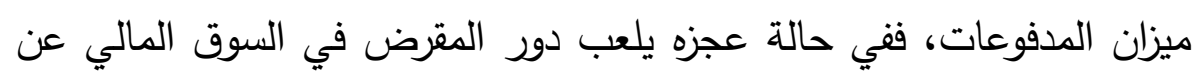

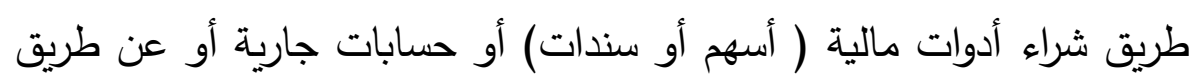

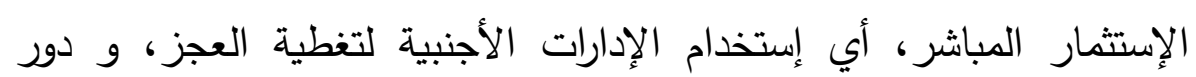

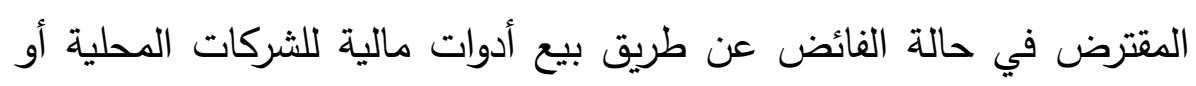
توظيف هذا الفائض في الأسواق المالية الأجنبية.

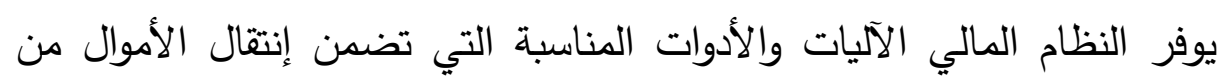

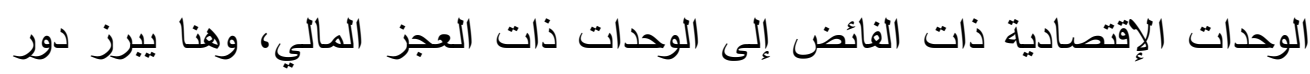

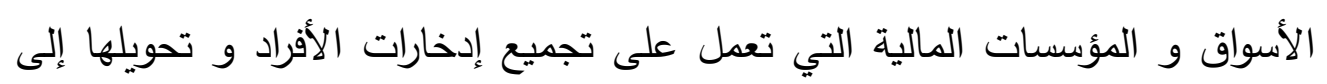
إستثمارات فعلية تؤدي إلى زيادة الإنتاج والدخل وإستخدام القوى العاملة.

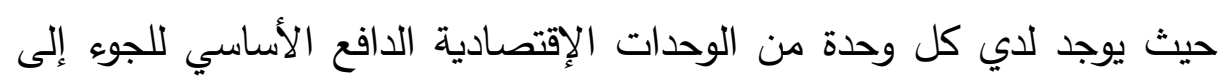

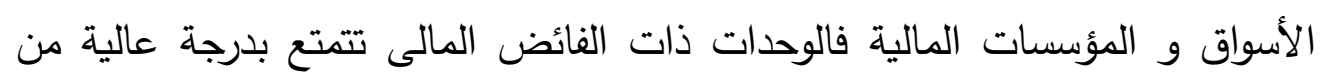

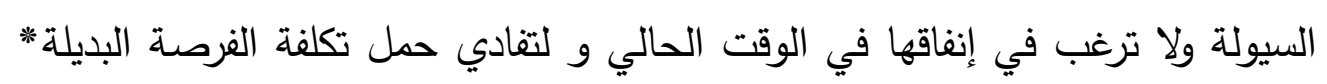

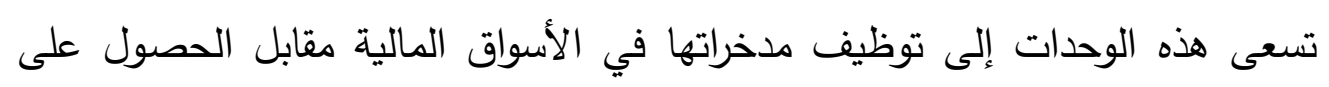

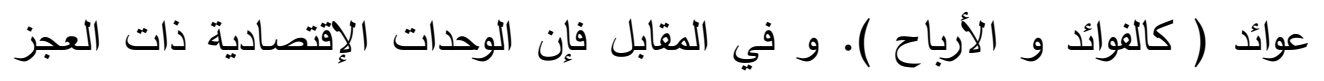

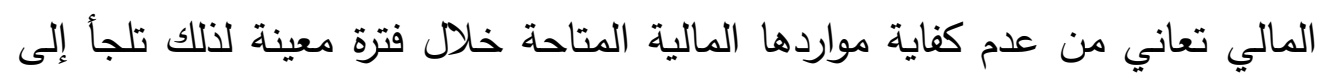

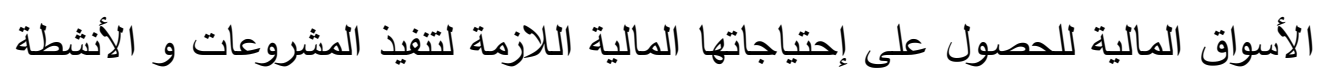

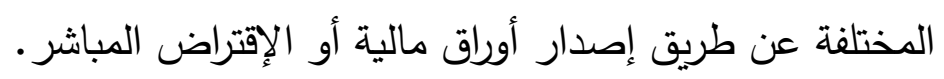

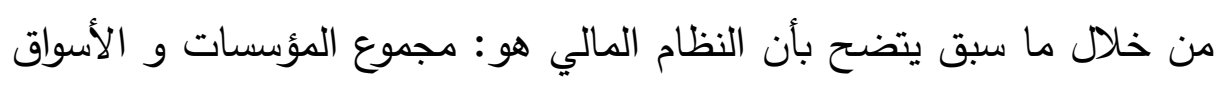
و القنوات التي توفر الآلية الضرورية لخلق و تبادل الأصول المالية بين الوحدات 
الإقتصادية ذات القدرة التمويلية و الوحدات الإقتصادية العجزية، هذه العملية التي يتحقق من خلالها توظيف الأموال وزيادة الفرص الإستثمارية، و بدون وجود هذه القناة

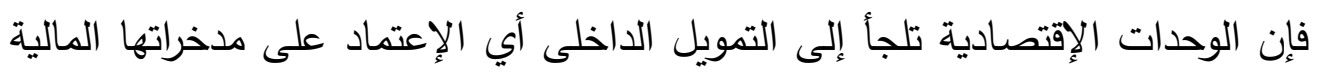

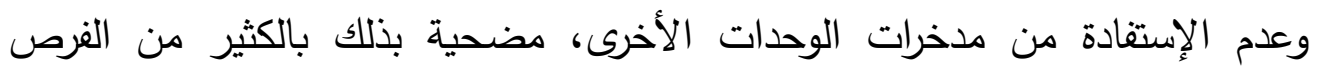
الإستثمارية، و هذا يتسبب في حالة من الركود و إنخفاض الإنتاج و الاخل و تزايد البطالة.

r- مقدمو الخدمات المالية: ( الأسواق المالية بالإضافة إلى مجموع المؤسسات (المالية): (- n) في معظم البلدان توجد إلى جانب البنك المركزي خمسة أنواع رئيسية من

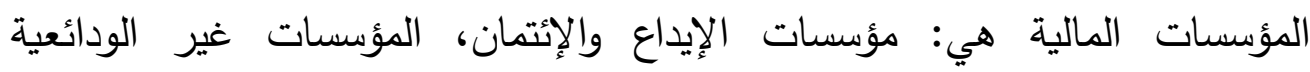

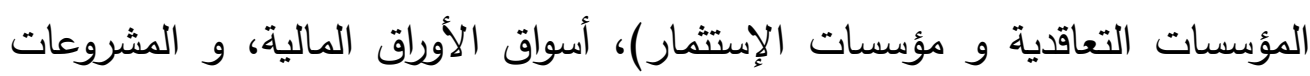

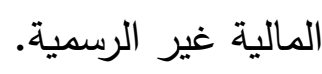
المطلب الثاني: مفهوم النمو الاقتصادي: الأول: تعريف النمو الاقتصادي: حظي موضوع النمو الاقتصادي بالكثير من الاهتمام من قبل العلماء والمفكرين،

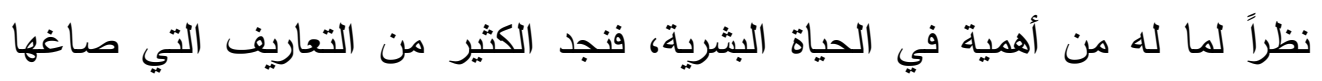

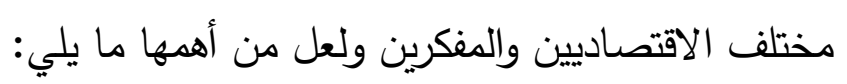
يقول Francois Perroux يمثل النمو الاقتصادي الزيادة المستمرة خلال فترة أو عدة فترات طويلة لمؤشر الإنتاج بالحجم لبلد ما، الناتج الإجمالي الصافي بالقيمة لئل الحقيقية.

يعرف Joseph Schumpeter النمو الاقتصادي بأنه التغير البطيء على المدى الطويل، والذي يتم من خلاله الزيادة التدريجية والمستمرة في معدل نمو السكان

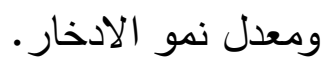


حسب Simon Kuznets يعرف النمو الاقتصادي لبلد ما على أنه زيادة طويلة

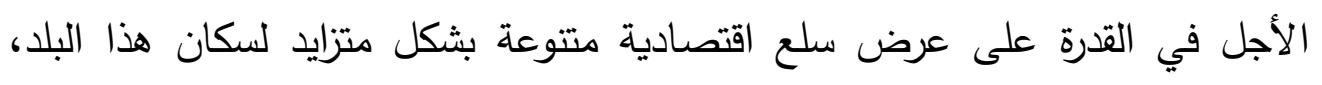
تستند هذه القدرة المتنامية على التقدم التكنولوجي التنظيمات المؤسساتية والإيديولوجية التي تتطلبها.

ومن وجهة نظر Dominick Salvatore يمكن تعريف النمو الاقتصادي على

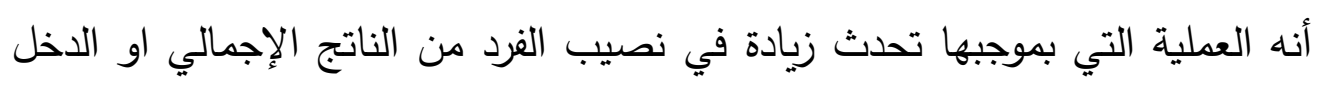
الحقيقي لبلد ما على فترة طويلة من الزمن من خلال الزيادة المستمرة في الإنتاجية

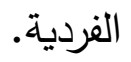

ويذهب Milton Friedman أن النمو الاقتصادي إنما يعني توسع الجهاز الإنتاجي في اتجاه أو أكثر، بدون تغيرات في الهيكل الاقتصادية.

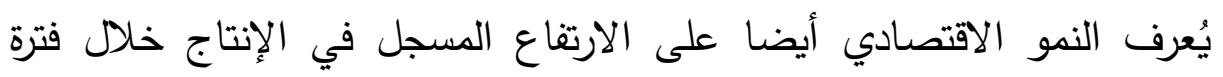

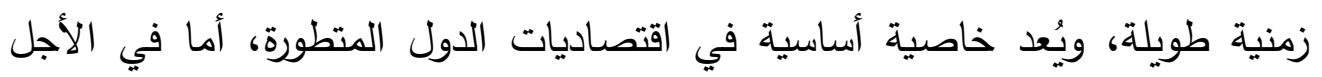
القصير والمتوسط فالاقتصاديون يستخدمون مصطلح رواج والذي يعبر عن مرحلة

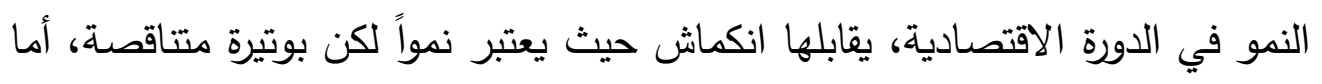
الركود فيعني تراجع ويصبح معدل النمو الاقتصادي سالباً . نتائج الاراسة:

\section{اولأ: خطوات تقدير نموذج الاراسة:}

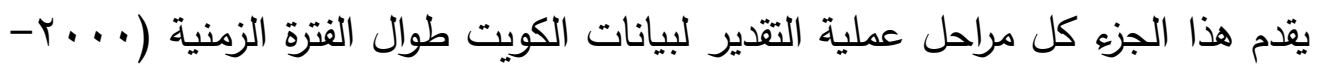

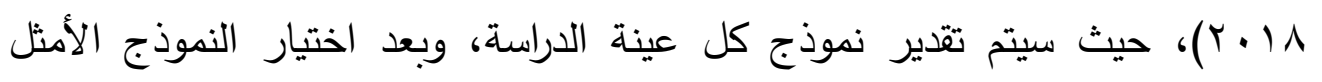

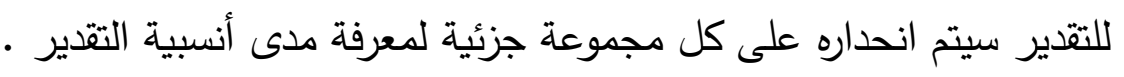

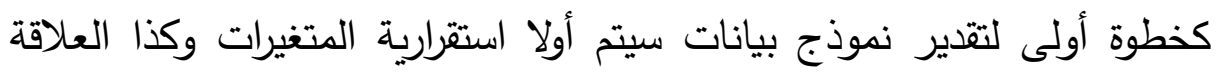

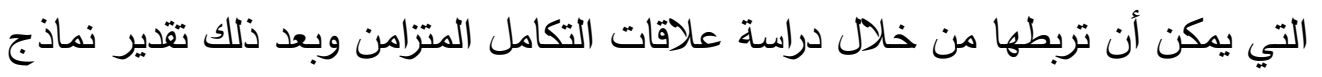

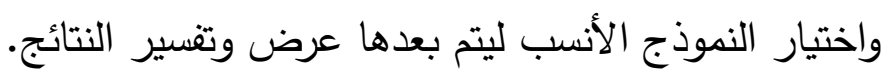


نقوم في هذا العنصر بتقديم بعض تعاريف الإحصاء الوصفي لمتغيرات العينة

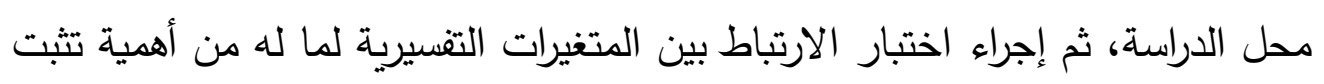

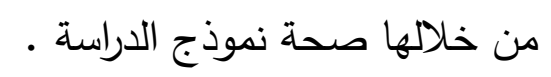
GDP

•DC الائتمان المحلي المقدم إلى القطاع الخاص نسبة إلى الناتج الإجمالي المحلي.

• BM المعروض النقدي نسبة إلى اجمالي الناتج : • • Cl النفقات النهائية للاستهلاك نسبة إلى الناتج الإجمالي المحلي.

$$
\begin{array}{r}
\text { TO : معامل الانفتاح التجاري. SO } \\
\text { :INF }
\end{array}
$$

\section{ثانيا: التحليل الوصفي للمتغيرات:}

استخدمت الدراسة العديد من الأساليب الإحصائية الوصفية من أجل وصف متغيرات النموذج القياسي، من خلال مقاييس التثتت والنزعة المركزية، حيث تم استخدام الوسط الحسابي و الوسيط والانحراف المعياري، لكل متغيرمن متغيرات النمودج القياسي، والجدول (1) يوضح أهم المقاييس الإحصائية لكل من نصيب الفرد من الناتج المحلي الإجمالي، الائتمان المحلي المقدم إلى القطاع الخاص نسبة إلى الناتج الإجمالي المحلي، المعروض النقدي نسبة إلى اجمالي الناتج، النفقات النهائية للاستهلاك نسبة إلى الناتج الإجمالي المحلي، معامل الانفتاح التجاري، معدل التضخم

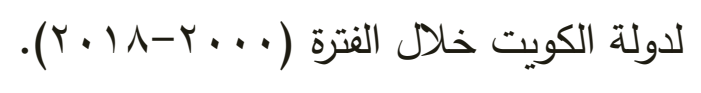


جدول رقم (1) تطور نصيب الفرد من الناتج المحلي الاجمالي والمتغيرات المستقلة

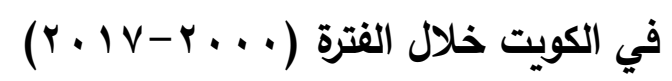

\begin{tabular}{|c|c|c|c|c|c|c|}
\hline معدل التضخم & معامل الانفتاح & للاستنهلاك اللهائية & الموسع التي النقي & 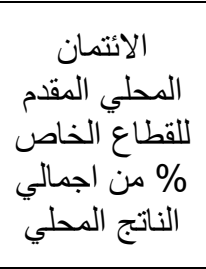 & اجمالي الناتج & السنوات \\
\hline 1.81 & 71.94 & 71.63 & 70.55 & 45.39 & 18440.4 & 2000 \\
\hline 1.3 & 70.76 & 81.15 & 86.05 & 56.62 & 16587.2 & 2001 \\
\hline 0.88 & 69.26 & 75.38 & 83.22 & 58.33 & 17846.3 & 2002 \\
\hline 0.96 & 76.52 & 57.27 & 70.55 & 59.46 & 22148.4 & 2003 \\
\hline 1.24 & 70.04 & 47.86 & 86.05 & 56.36 & 27011.7 & 2004 \\
\hline 4.14 & 75.62 & 42.46 & 83.22 & 50.93 & 35591 & 2005 \\
\hline 3.05 & 72.56 & 43.94 & 72.9 & 49.95 & 42781.4 & 2006 \\
\hline 5.5 & 73.16 & 41.12 & 66.7 & 59.57 & 45782.3 & 2007 \\
\hline 10.6 & 75.12 & 57.74 & 55.46 & 57.26 & 55494.9 & 2008 \\
\hline 4.6 & 64.52 & 47.12 & 54.02 & 79.11 & 37561.6 & 2009 \\
\hline 4.5 & 86.361 & 38.23 & 58.19 & 74.3 & 38577.5 & 2010 \\
\hline 4.8 & 95.06 & 37.28 & 65.26 & 60.8 & 48631.7 & 2011 \\
\hline 3.25 & 93.50 & 41.53 & 60.63 & 55.74 & 51979.1 & 2012 \\
\hline 2.7 & 97.60 & 47.58 & 65.64 & 59.57 & 49388.1 & 2013 \\
\hline 2.9 & 99.14 & 65.87 & 72.63 & 67.64 & 44062.3 & 2014 \\
\hline 3.2 & 99.64 & 70.93 & 99.32 & 98.51 & 29869.5 & 2015 \\
\hline 3.1 & 96.15 & 68.60 & 106.79 & 103.77 & 27653 & 2016 \\
\hline 2.2 & 98.52 & 69.85 & 101.9 & 99.24 & 29759 & 2017 \\
\hline 0.54 & 97.5 & 64.19 & 101.5 & 89.3 & 33994 & 2018 \\
\hline 3.22 & 83.31 & 56.3 & 76.87 & 67.46 & 35429.44 & المتوسط \\
\hline
\end{tabular}

المصدر: قاعدة بيانات ،البنك الدولي 
تثير بيانات الجدول (Y) أن المتوسط الحسابي نصيب الفرد من الناتج المحلي

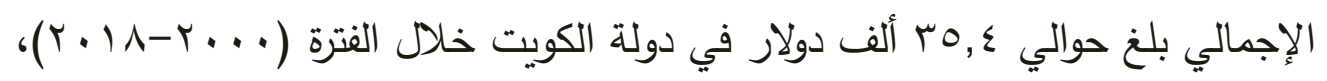
كما بلغت ادني قيمة 7,7 ألف دولار ، وأعلي قيمة 00,0 ألف دولار، أما بالنسبة الائتمان المحلي المقدم إلى القطاع الخاص نسبة إلى الناتج الإجمالي المحلي في دولة

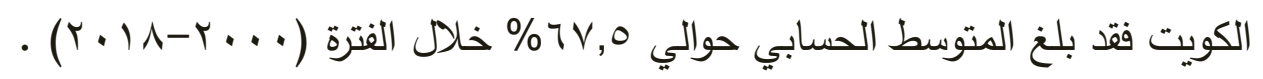
الجدول رقم (ץ) المقاييس الاحصائية الوصفية لمتغيرات النموذج القياسي

\begin{tabular}{|c|c|c|c|c|c||}
\hline & Mean & Median & $\begin{array}{c}\text { Std. } \\
\text { Dev }\end{array}$ & Maximum & Minimum \\
\hline \hline GDP & 35429 & 35591 & 12068 & 55500 & 16600 \\
\hline DC & 67.5 & 59.6 & 1.79 & 103.8 & 45.4 \\
\hline BM & 76.5 & 3.8 & 1.65 & 106.8 & 54.02 \\
\hline CI & 56.3 & 57.2 & 1.42 & 81.1 & 37.2 \\
\hline TO & 83.3 & 76.5 & 1.28 & 99.6 & 64.5 \\
\hline INF & 2.22 & 3.05 & 2.30 & 10.6 & 0.54 \\
\hline
\end{tabular}

المصدر : حسبت من بيانات جدول رقم (1)

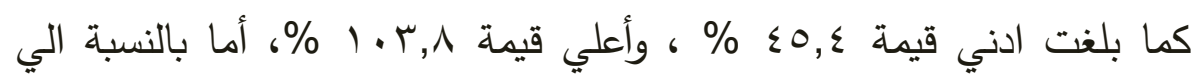

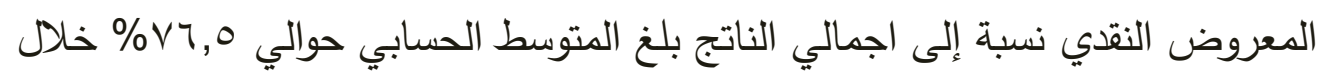

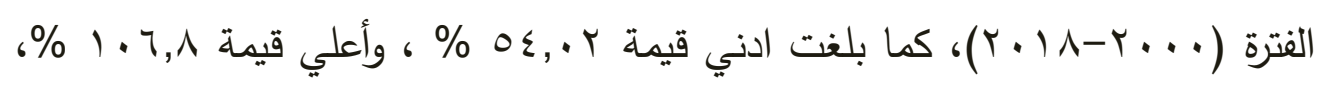
أما بالنسبة الي النفقات النهائية للاستهلاك نسبة إلى الناتج الإجمالي المحلي بلغ 


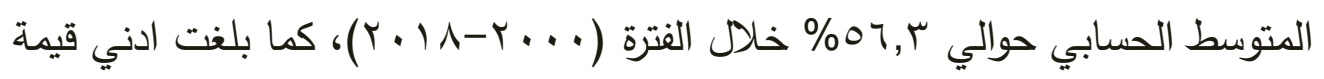

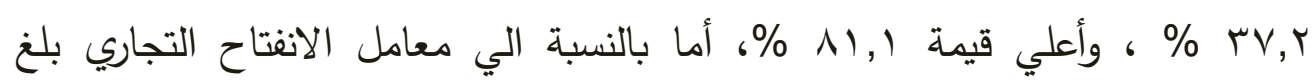

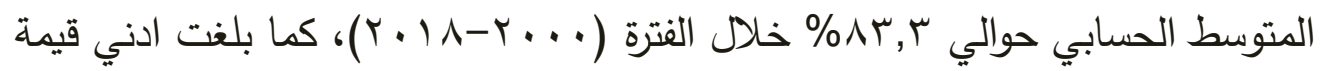

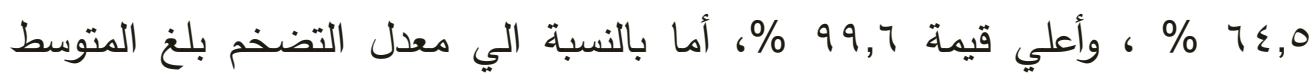

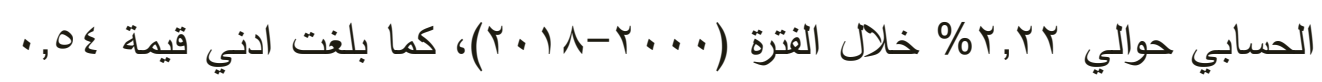

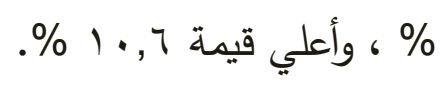

ثانيا:الارتباط بين متغيرات الاراسة:

يساعد فحص الارتباط بين المتغيرات التقسيرية من التأكد من خلو النموذج من أهم المشاكل التي يمكن أن تحدث عند تقدير نموذج بيانات بانيل، بحيث أن معاملات الارتباط المتعدد تكون ذات صلة بالانحدار الخاص بكل متغير مستقل بالنسبة لباقي

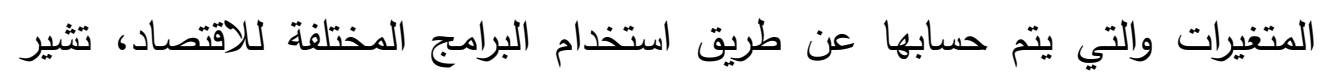

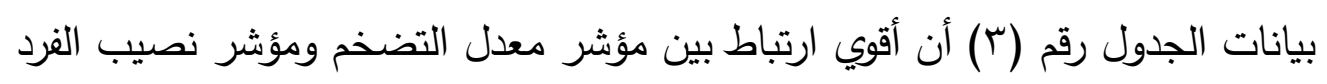
من الناتج المحلي حيث بلغ 9.9\%٪، يليه الارتباط بين مؤشر الائتمان المحلي المقدم

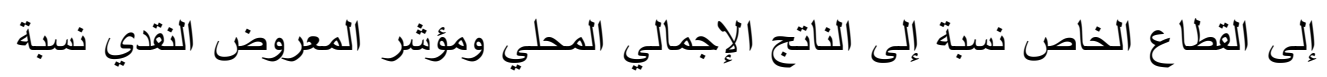

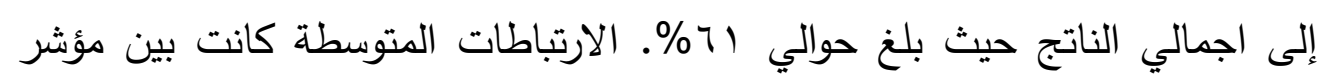
الائتمان المحلي المقدم إلى القطاع الخاص نسبة إلى الناتج الإجمالي المحلي ومؤشر معامل الانفتاح التجاري حيث بلغ حوالي ه\%٪. أما الارتباطات السلبية فكان أهمها بين مؤشر المعروض النقدي نسبة إلى اجمالي الناتج ومؤشر معدل التضخم بنسبة r\%\% . وبين مؤشر النفقات النهائية للاستهلاك نسبة إلى الناتج الإجمالي المحلي

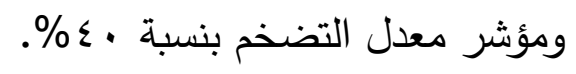




\section{الجدول رقم (ץ) مصفوفة الارتباط لمتغيرات الدراسة}

\begin{tabular}{|c|c|c|c|c|c|c|}
\hline & GDP & DC & BM & Cl & TO & INF \\
\hline \hline GDP & 1 & - & -0.553 & -0.681 & 0.308 & 0.695 \\
\hline DC & -0.125 & 1 & 0.610 & 0.326 & 0.591 & -0.094 \\
\hline BM & -0.553 & 0.610 & 1 & 0.601 & 0.361 & -0.531 \\
\hline CI & -0.681 & 0.326 & 0.601 & 1 & 0.066 & -0.400 \\
\hline TO & 0.308 & 0.591 & 0.361 & 0.066 & 1 & -0.085 \\
\hline INF & 0.695 & - & -0.531 & -0.400 & -0.085 & 1 \\
\hline
\end{tabular}

المصدر : حسبت من بيانات جدول رقم (1)

ثالثا: دراسة إستقرارية السلاسل الزمنية للمتغرات موضع الدراسة:

يعتمد صحة تقدير النماذج القياسية علي افتراض توافر الخصائص الإحصائية

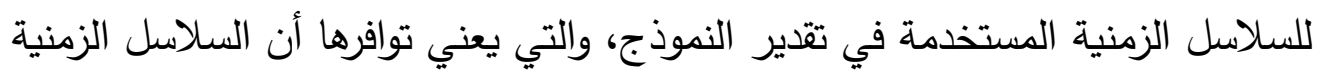

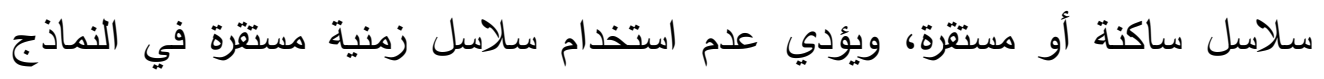

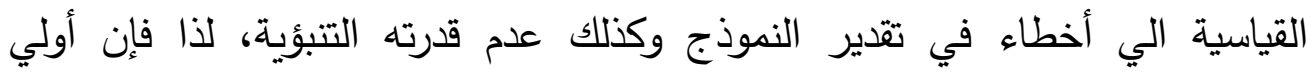
خطوات تقدير النموذج القياسي هو الكثف عن مدي غستقرار وسكون السلاسل الزمنية

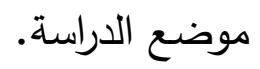
ستعتمد الدراسة للكثف عن إستقرار وسكون السلاسل الزمنية علي إختبارات

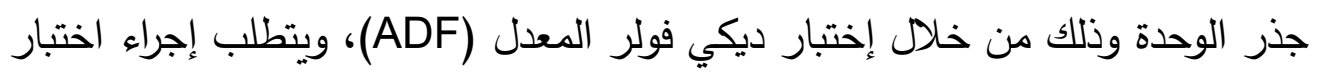


استقرار السلاسل الزمنية الاعتماد علي تحديد فترات الإبطاء الزمني حيث تم الاعتماد

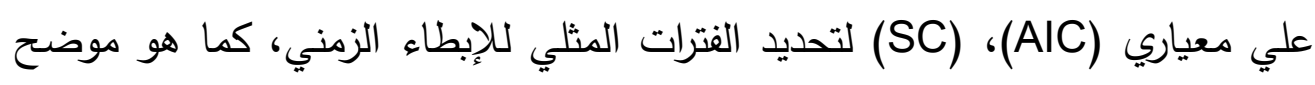

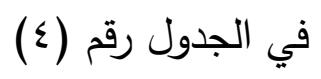

أ- اختبار ديكي - فولر الموسع (ADF):

وبإستعراض النتائج الواردة في الجدول رقم (؛) والذي يوضح نتائج اختبار جذر

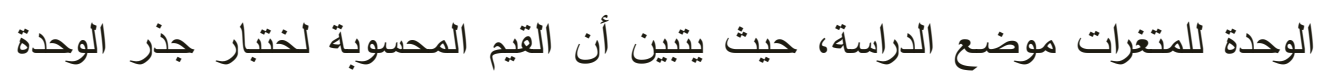

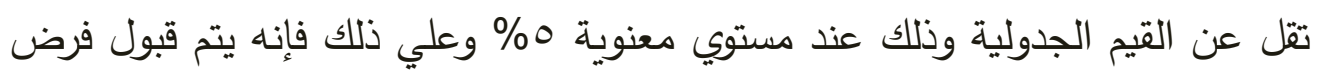
العدم الذي يثير الي وجود جذر الوحدة بالسلاسل الزمنية للمتغيرات جميعها موضع مهيع الدراسة، وهو ما يعني أن تلك الكتغيرات غير ساكنة في المستوي. جدول رقم ( ؛): عدد فترات الإبطاء الزمني للمتغيرات موضع الدراسة وفقا لنموذج الاندار الذاتي

\begin{tabular}{|c|c|c|c|c|c|c||}
\hline \multirow{2}{*}{} & \multicolumn{3}{|c|}{ AIC } & \multicolumn{3}{c||}{ SC } \\
\cline { 2 - 7 } & Zero & 1 & 2 & Zero & 1 & 2 \\
\hline GDP & 21.48 & $20.67^{*}$ & 20.71 & 21.53 & $20.77^{*}$ & 20.85 \\
\hline DC & 8.66 & $7.74^{*}$ & 7.75 & 8.71 & $7.84^{*}$ & 7.90 \\
\hline BM & 8.60 & $7.59^{*}$ & 7.63 & 8.65 & $7.69^{*}$ & 7.77 \\
\hline CI & 8.01 & 7.23 & $7.10^{*}$ & 8.06 & 7.33 & $7.25^{*}$ \\
\hline TO & 8 & $6.85^{*}$ & 6.91 & 8.05 & 6.95 & 7.06 \\
\hline INF & 4.61 & $4.41^{*}$ & 4.53 & 4.66 & $4.51^{*}$ & 4.68 \\
\hline
\end{tabular}


ويتطلب تحويل السلاسل الزمنية الي سلاسل ساكنة إجراء الفروق الأولي

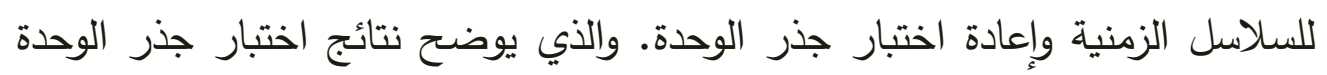

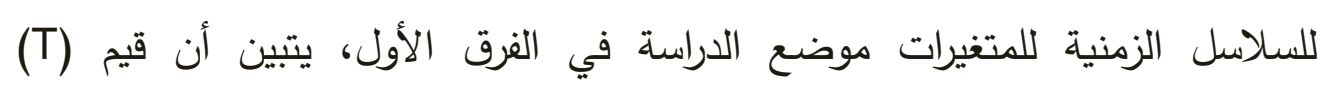

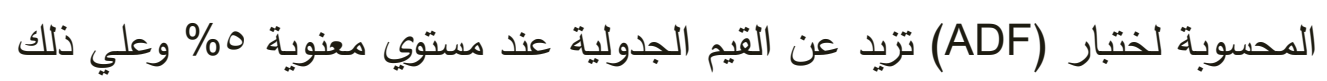
فإنه يتم رفض فرض العدم الذي يشير الي وجود جذر الوحدة بالسلاسل الزمنية

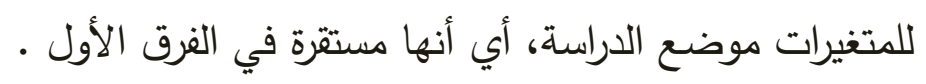
الجدول (•) نتائج اختبارات جذر الوحدة لسكون السلاسل الزمنية

\begin{tabular}{|c|c|c|c|c|}
\hline \multicolumn{3}{|c|}{$\mathrm{ADF}$} & \multirow{2}{*}{ الوحدة جذر } & \multirow[t]{2}{*}{ الدتغيرات } \\
\hline الاحتمالية & \% معلمة الاختبار & معملة الاختبار & & \\
\hline 0.397 & $\begin{array}{l}-3.04 \\
\end{array}$ & $\begin{array}{l}-1.73 \\
\end{array}$ & Level & \multirow[t]{2}{*}{ GDP } \\
\hline 0.028 & -3.05 & -3.35 & Deff 1st & \\
\hline 0.599 & -3.04 & -1.31 & Level & \multirow[t]{2}{*}{$\mathrm{DC}$} \\
\hline 0.039 & -3.05 & -3.18 & Deff 1st & \\
\hline 0.764 & -3.04 & -0.90 & Level & \multirow[t]{2}{*}{$\mathrm{BM}$} \\
\hline 0.018 & -3.05 & -3.56 & Deff 1st & \\
\hline 0.086 & -3.05 & -2.74 & Level & \multirow[t]{2}{*}{$\mathrm{CI}$} \\
\hline 0.041 & -3.05 & -3.14 & Deff 1st & \\
\hline 0.724 & -3.04 & -1.01 & Level & \multirow[t]{2}{*}{ OT } \\
\hline 0.0007 & -3.05 & -5.26 & Deff 1st & \\
\hline 0.259 & -3.04 & -2.06 & Level & \multirow[t]{2}{*}{ INF } \\
\hline 0.001 & -3.05 & -4.91 & Deff 1st & \\
\hline
\end{tabular}

المصدر : اعتمادا علي برنامج Eviews. 


\section{رابعا: تقدير واختبار النموذج القياسي:}

سيتم استخدام أسلوب الانحدار المتعدد الخطي في تقدير النموذج القياسي

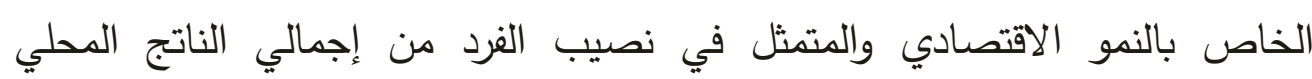
ولمعرفة الصيغة الرياضية المناسبة لتقديره :

$\mathrm{GDP}=\mathrm{B} 0+\mathrm{B} 1 \mathrm{DC}+\mathrm{B} 2 \mathrm{BM}+\mathrm{B} 3 \mathrm{Cl}+\mathrm{B} 4 \mathrm{TO}+\mathrm{B} 5 \mathrm{INF}$

الجدول رقم (†) نتائج تقدير المتغيرات المستقلة المؤثر علي نصيب الفرد من الناتج

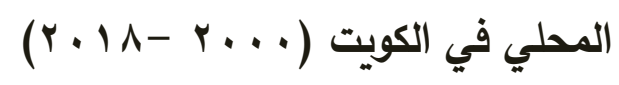

\begin{tabular}{|c|c|c|c|c|}
\hline نوع الدالة & النموذج & $\mathrm{R} 2$ & $\mathrm{R}-2$ & $\mathrm{~F}$ \\
\hline خطي & 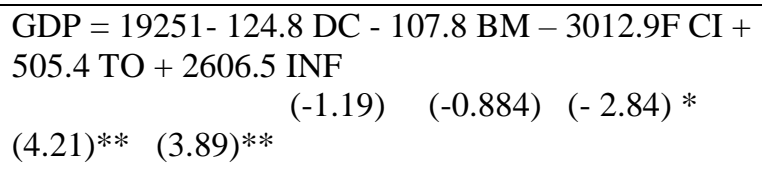 & 0.86 & 0.81 & $17.1 * *$ \\
\hline لوغاريتمي & $\begin{array}{l}\mathrm{GDP}=4.06-0.06 \mathrm{Log} \mathrm{DC}-0.29 \log \mathrm{BM}-0.56 \\
\log \mathrm{CI}+1.03 \log \mathrm{TO}+0.25 \mathrm{INF} \\
(2.90)^{*}(2.71)\end{array}$ & 0.82 & 0.75 & 12.2 \\
\hline
\end{tabular}

المصدر : حسبت من الجدول رقم (')

تثير بيانات الجدول رقم (†) تأكد إحصائيا معنوية النموذج الخطي الكامل

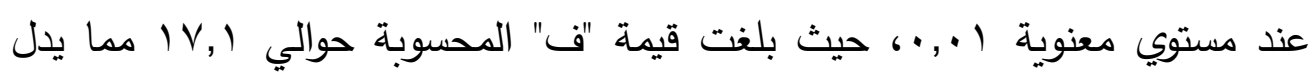
علي كفاءة الدالة المقدرة في التعبير بدقة عن العلاقة بين المتغيرات المستقلة نصيب لهبه

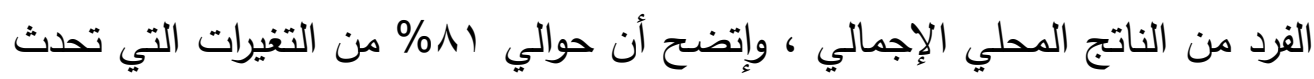

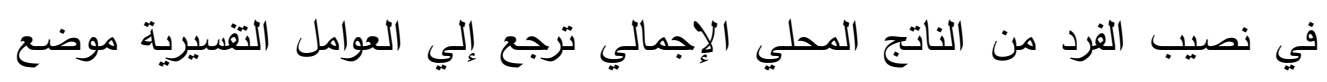

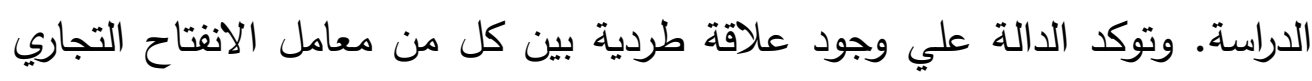

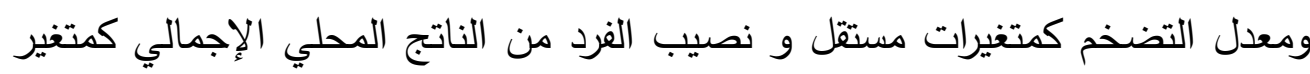

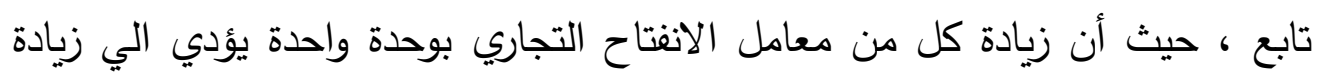

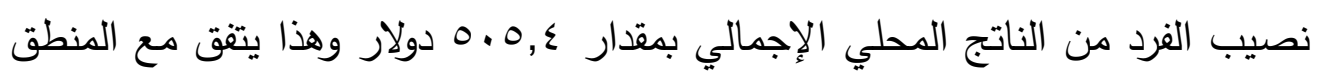


الاقتصادي، في حين يرتط معدل التضخم بعلاقة طرديا مع نصيب الفرد الناتج المحلي الإجمالي حيث ان زيادة معدل التضخم بوحدة واحد يؤدي الي زيادة نصيب الفرد الناتج المحلي الإجمالي بمقدار T ـ بr دولار وهذا لايتقق مع المنطق الاقتصادي .بينما لم تثبت معنوية كل من الائتمان المحلي المقدم إلى القطاع الخاص نسبة إلى الناتج الإجمالي المحلي و المعروض النقدي نسبة إلى اجمالي الناتج و النفقات النهائية للاستهلاك نسبة إلى الناتج الإجمالي المحلي. 


\section{المــراجـع}

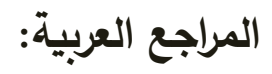

ا. عبد الغفار حنفي ، رسمية قرياقص " أسواق المال و تمويل المشروعات "

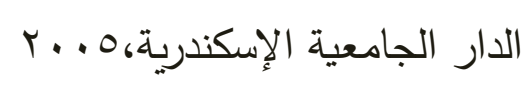

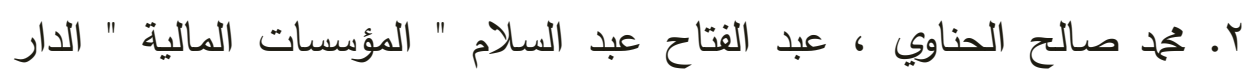

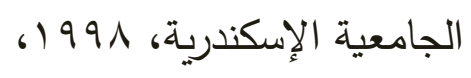

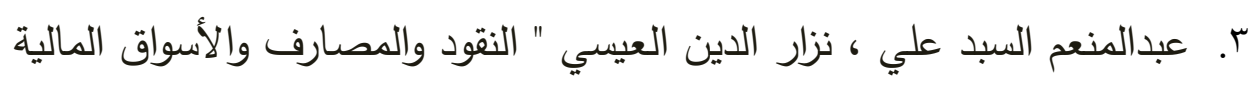

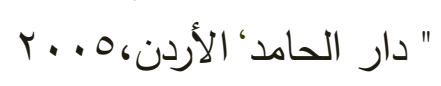

ء. عبد الله الصعيدي، مبادئ علم الاقتصاد، مطابع البيان التجارية، دبي،

$$
\text { .r. . E }
$$

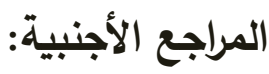

5. Paul Massé, Histoire économique et sociale du monde : de l'origine de l'humanité au XXe siècle, Tome 1, Editions I'harmattan, Paris, 2011,

6. David Edward O'connor, The Basics of Economics, Greenwood Publishing Group, Inc, USA, 2004.

7. Fidelis Ezeala-Harrison, Economic Development: Theory and Policy Applications, Greenwood Publishing Group, Inc, USA, 1996. 